\title{
Uso eficiente do canal em comunicações full-duplex através de uma reserva de canal inovadora
}

\author{
Lucas de Melo Guimarães ${ }^{1}$, Jacir Luiz Bordim ${ }^{1}$ \\ ${ }^{1}$ Departamento de Ciência da Computação - Universidade de Brasília (UnB) \\ 70.910-900 - Brasília - DF - Brazil \\ lucasmg@aluno.unb.br, bordimeunb.br
}

\begin{abstract}
Next generation (5G) mobile wireless networks are required to satisfy increasing demands of throughput in dense environments. Full-duplex (FD) communications may allow to overcome these requirements, once they are expected to improve throughput and channel use. In order to better exploit the potential of FD, MAC protocols have to be designed taking advantage of $F D$ particular characteristics. However, several of existing FD MAC protocols are based on IEEE 802.11, which was designed for half-duplex communications. In this context, our work proposes FDMR-MAC (Full-duplex Multiple Receiver $M A C)$. FDMR-MAC introduces an innovative channel reservation technique in order to enhance channel use and throughput. FDMR-MAC is compared with state-of-art MAC protocols through an extension of a well-known model based on stochastic processes. The results of this evaluation indicate that FDMR-MAC surpasses state-of-art protocolos in all evaluated cases. The throughput improvement reached up to $67 \%$.
\end{abstract}

Resumo. A próxima geração $(5 G)$ de redes móveis é necessária para satisfazer os crescentes requisitos de vazão em redes cada vez mais densas. As comunicações full-duplex (FD) podem ajudar a cumprir estes requisitos, pois espera-se que elas melhorem a vazão e o uso do canal. Para melhor aproveitar o potencial de FD, as técnicas de controle de acesso ao meio (MAC) devem ser projetadas para tirar vantagem das características de FD. Porém, diversos mecanismos MAC para FD baseiam-se no padrão IEEE 802.11 que fora projetado para comunicações half-duplex. Neste contexto, o presente trabalho propõe o FDMR-MAC (Full-duplex Multiple Receiver MAC). O FDMR-MAC recorre a um mecanismo inovador de reserva de canal para possibilitar melhor uso do canal e maior vazão. O FDMR-MAC é avaliado em comparação com técnicas MAC do estado da arte por meio da extensão de um modelo matemático muito utilizado e baseado em processos estocásticos. Os resultados apontam que o FDMR-MAC supera as demais técnicas avaliadas em todos cenários considerados. A melhoria alcança até $67 \%$ em termos de vazão.

\section{Introdução}

As comunicações full-duplex (FD) permitem que um nó transmita e receba dados simultaneamente em um mesmo canal [Amjad et al. 2017]. O principal problema de utilizar comunicações full-duplex é a auto-interferência, isto é, a interferência 
percebida na antena quando esta está em um mesmo momento transmitindo e recebendo algum sinal [Zhang et al. 2015] [Tang et al. 2016] [Zhang et al. 2016]. Entretanto, o desenvolvimento de diversas técnicas para contornar as limitações impostas pela auto-interferência fomentou o desenvolvimento das comunicações fullduplex [Thilina et al. 2015] [Zhang et al. 2015]. Quando comparadas às comunicações half-duplex, as comunicações full-duplex aumentam a capacidade da rede, diminuem as perdas de dados e aumentam o uso do canal [Thilina et al. 2015] [Amjad et al. 2017]. No entanto, explorar os benefícios das comunicações full-duplex não é uma tarefa trivial e requer o projeto de técnicas de controle de acesso ao meio (MAC) específicas para este tipo de comunicação. Tais técnicas devem levar em consideração as particularidades das comunicações full-duplex para melhor explorar seu potencial para que aconteça um eficiente gerenciamento dos recursos da rede [Thilina et al. 2015].

Vários protocolos de controle de acesso ao meio foram pro-
jetados para comunicações full-duplex, tais como os apresentados
em [Zhang et al. 2015] [Marlali, Deniz and Gurbuz, Ozgur 2017]. Um dos protocolos mais proeminentes para comunicações full-duplex é o FD-MAC (Full-duplex MAC) [Zhang et al. 2015]. Ele propõe um mecanismo de reserva de canal permitindo uma operação sem colisões em um cenário onde o nó emissor é capaz de enviar dados ao destinatário, enquanto o destinatário envia dados de volta ao emissor. Assim, o FD-MAC melhora a vazão da rede quando comparado com técnicas de controle de acesso ao meio baseadas em comunicações half-duplex. Além do mais, os autores [Zhang et al. 2015] apresentaram discussões interessantes no que se refere à alocação de potência e atenuação dos efeitos causados pela auto-interferência.

Porém, tanto o FD-MAC quanto os outros mecanismos de controle de acesso ao meio projetados para comunicações full-duplex simplesmente se baseiam em algumas das técnicas propostas pelo padrão IEEE 802.11 [IEEE 2007], tais como o uso do algoritmo de backoff exponencial e a realização de reserva de canal via quadros RTS/CTS (Request to Send/Clear to Send) acoplada à detecção de portadora virtual via NAV (Network Allocation Vector) para evitar colisões. Embora os quadros RTS/CTS introduzam considerável latência à rede, seu uso é amplamente realizado em comunicações half-duplex devido ao fato dele minimizar os efeitos do problema do terminal escondido [Guimarães and Bordim 2018a]. Portanto, vários protocolos MAC recorrem aos quadros RTS/CTS para mitigar colisões, como ocorre em [Zhang et al. 2015] [Al-Kadri et al. 2016] e [Garcia-Luna-Aceves, JJ 2017]. Por exemplo, o FD-MAC [Zhang et al. 2015] considera o uso de um handshake de três vias com o auxílio dos quadros RTS e FCTS (Full-duplex Clear to Send). O FCTS inclui um campo extra em comparação com o CTS tradicional do padrão IEEE 802.11. Este campo extra está relacionado ao tempo de transmissão dos dados. A informação contida no FCTS é necessária para sincronizar os nós da rede para evitar colisões. No entanto, é importante ponderar que o padrão IEEE 802.11 foi projetado para comunicações halfduplex. Portanto, técnicas baseadas no referido padrão podem não ser capazes de explorar de modo apropriado o potencial decorrente da utilização de comunicações full-duplex. As melhorias relacionadas à vazão dos protocolos de controle de acesso ao meio projetados para comunicações full-duplex do estado da arte vem principalmente da sua política de alocação de energia e do uso propriamente dito de comunicações full-duplex. 
Para melhor explorar os benefícios das comunicações full-duplex, o mecanismo FDDS-MAC (Full-Duplex Dynamic Scheduling MAC) foi proposto por [Guimarães and Bordim 2018b]. O FDDS-MAC foi projetado com o intuito de melhorar o uso do canal. Para tanto, o FDDS-MAC inclui uma fase de decisão anterior à reserva de canal. A fase de decisão visa selecionar um nó destino de modo a estabelecer o par de nós emissor/destinatário tal que este par maximize o uso do canal. A fase de decisão do FDDS-MAC em conjunto com uma política de escalonamento tendem a melhorar tanto a vazão quanto o uso do espectro. Embora o FDDS-MAC seja capaz de obter relevantes melhorias no que diz respeito à vazão quando comparado ao FD-MAC, a eficiência do FDDS-MAC está ligada a eficiência da fase de decisão. Esta fase extra entretanto introduz latência à comunicação e pode se tornar crítica em alguns cenários, como apontado por [Guimarães and Bordim 2018b].

Portanto, este trabalho propõe a técnica de controle de acesso ao meio FDMRMAC (Full-duplex Multiple Receiver MAC). O FDMR-MAC tem por objetivo aumentar a probabilidade de se estabelecer uma comunicação na qual o par de nós emissor/destinatário possuem dados para transmitir um para o outro. O FDMR-MAC realiza isso por meio de um mecanismo inovador de reserva de canal sem recorrer a uma fase de decisão como o FDDS-MAC. Assim, o FDMR-MAC reduz a latência introduzida pelo FDDS-MAC melhorando assim a vazão. Outra contribuição notável deste trabalho é a extensão de um modelo matemático para calcular a vazão no contexto de comunicações full-duplex. A partir deste modelo, foram feitas avaliações que indicam que o FDMRMAC possui ganhos de vazão em todos os cenários avaliados quando comparado com o FD-MAC e o FDDS-MAC. Resultados analíticos mostram que o ganho do FDMR-MAC em termos de vazão chegou a alcançar $67 \%$.

O restante do trabalho se encontra organizado da seguinte forma: Seção 2 apresenta a técnica de controle de acesso ao meio (FDMR-MAC) proposta neste trabalho, bem como suas principais características, motivações e seus objetivos; Seção 3 expõe diversas avaliações realizadas contrapondo a técnica de controle de acesso ao meio proposta com outras existentes do estado da arte; Finalmente, a Seção 4 conclui este trabalho indicando tópicos e rumos interessantes para trabalhos futuros.

\section{FDMR-MAC: Reserva de canal}

Esta seção apresenta a técnica de controle de acesso ao meio proposta neste trabalho no contexto de comunicações full-duplex. A referida técnica é denominada Full-Duplex Multiple Receiver MAC (FDMR-MAC). O principal objetivo do FDMR-MAC é maximizar a utilização do canal e a vazão em comunicações full-duplex. Para tanto, o FDMR-MAC recorre a um mecanismo inovador de reserva de canal que permite que um nó que vai iniciar uma comunicação (emissor) possa consultar alguns de seus vizinhos de modo a descobrir qual deles possui quadros de dados a serem enviados de volta ao nó emissor. Com base nesta informação, o nó emissor pode escolher estabelecer uma comunicação full-duplex com o nó que maximiza o uso do canal por ter tráfego de volta a ser enviado ao emissor. Assim, espera-se que o FDMR-MAC melhore o uso do canal e a vazão em redes que possuam a capacidade de operar com comunicações full-duplex.

Considere que $S$ seja o nó emissor e que $\mathcal{X}=\{B, C, D, E\}$ denota o conjunto de nós candidatos a serem escolhidos como destino de $S$, ou seja, o conjunto de nós que 
$S$ possui quadros de dados para enviar. Claramente, se $|\mathcal{X}|=1$, o nó $S$ possui quadros de dados para apenas um destino e a escolha do nó destino se torna trivial neste caso. Logo, suponha que $|\mathcal{X}|>1$. Neste caso, $S$ pode escolher o nó destino $R(R \in \mathcal{X})$, de tal modo que $R$ maximize o uso do canal. Para este fim, considera-se que um escalonamento de quadros de dados na fila do nó $S$ pode ser realizada com uma abordagem similar àquela descrita por [Guimarães et al. 2015]. É importante mencionar que o referido escalonamento não causa espera indefinida (starvation), uma vez que todos os nós disputam através do seus tempos de backoff o acesso ao canal para serem o nó emissor, de um modo semelhante ao descrito pelo padrão IEEE 802.11 [IEEE 2007].

Outro aspecto notável do FDMR-MAC está no fato de se recorrer ao uso de sinais pulso e tom para realizar a reserva de canal e a confirmação do recebimento dos quadros de dados (acknowledgment) de maneira eficiente. Os sinais pulso e tom auxiliam no processo de escolha do nó destino que maximiza a vazão da rede e o uso do canal. Os sinais pulso e tom podem ser transmitidos em menos tempo que os quadros (RTS/FCTS, ACK) [Shih et al. 2009]. Isso faz com que o uso destes sinais se torne interessante do ponto de vista de vazão, uma vez que ao se reduzir o tempo total da comunicação, se aumenta a vazão. Esta característica de ser menos oneroso que os quadros em termos de tempo de transmissão é exatamente o porquê do FDMR-MAC propor o uso de sinais pulso e tom em sua reserva de canal e no seu processo de escolha do nó destino. Como os referidos sinais são parte fundamental do FDMR-MAC, é necessário apresentar algumas considerações sobre estes.

\subsection{Considerações sobre sinais pulso e tom}

O tempo gasto para decodificar um sinal pulso/tom é definido por $T_{\text {sync }}$ e é considerado como sendo igual a $5 \mu \mathrm{s}$, uma vez que este é o valor apontado nas referências por [Shih et al. 2009]. Apesar de serem menos custosos em termos de tempo de transmissão que os quadros, os sinais pulso e tom são senoides e não carregam nenhum tipo de informação. Entretanto, uma comunicação necessita de informações contidas no cabeçalho MAC dos quadros, em especial, no que tange aos campos relacionados à origem, destino e duração da comunicação. Tais informações são consideradas como sendo codificadas nos sinais pulso e tom usando um mecanismo que possui probabilidade insignificante de falha. Por limitações de espaço, o referido mecanismo não será explicado em neste trabalho. Para maiores detalhes, pode-se consultar a descrição do mecanismo realizada por [Guimarães et al. 2015].

Considera-se ainda que sinais pulso e tom podem ser sobrepostos sem causarem colisão um ao outro, se eles forem transmitidos em diferentes sub-canais da banda (subcarriers) [Sen, Souvik and Roy Choudhury, Romit and Nelakuditi, Srihari 2011]. Seja $\theta$ o total de canais. Em um dado instante de tempo, um sinal pulso e tom pode ser decodificado corretamente se um sub-canal $F_{i}(0 \leq i \leq \theta)$ está tendo um sinal transmitido e seus sub-canais adjacentes $\left(F_{i-1}\right.$ e $\left.F_{i+1}\right)$ estão livres. Esta consideração é a mesma assumida por [Sen, Souvik and Roy Choudhury, Romit and Nelakuditi, Srihari 2011], uma vez que os sub-canais adjacentes podem causar interferências na transmissão em $F_{i}$ gerando possíveis perdas.

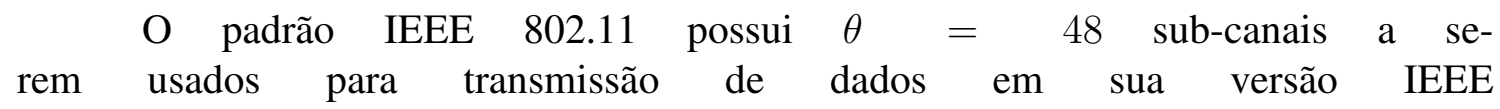



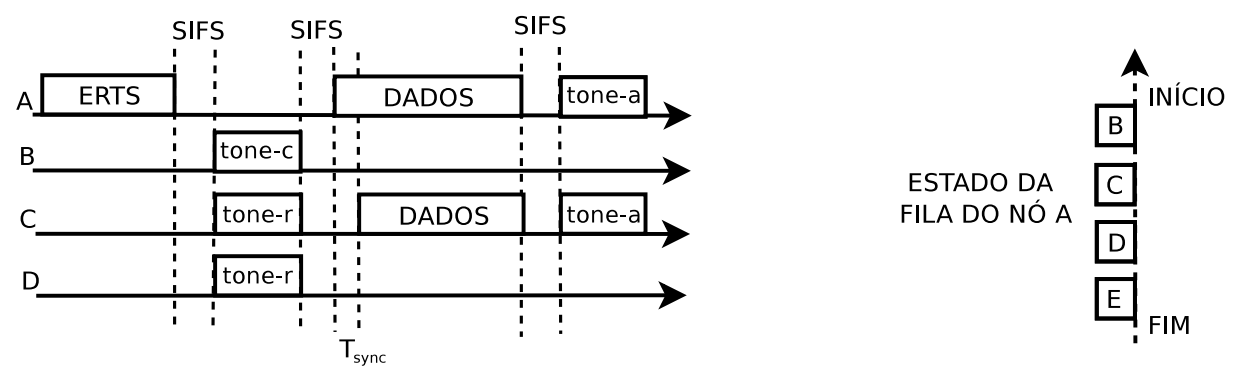

(a) Vizinhos possuem dados para o nó emissor.
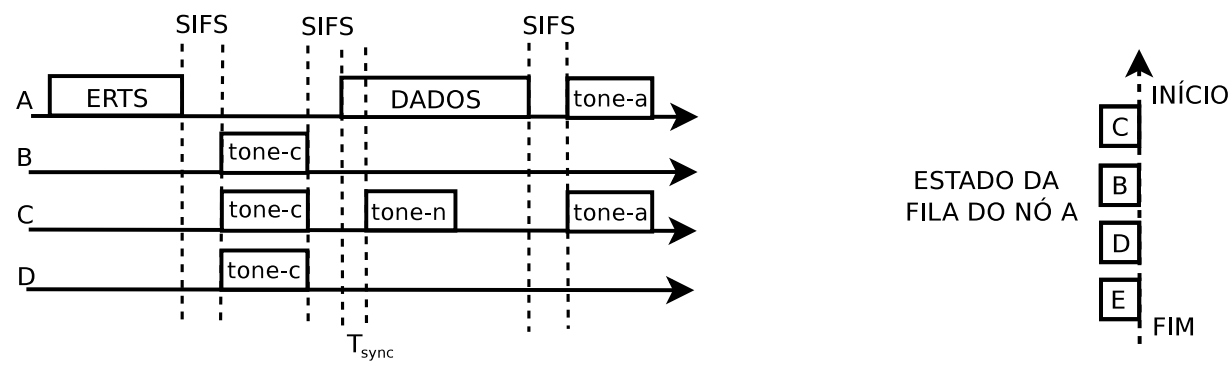

(b) Vizinhos não possuem dados para o nó emissor.

Figura 1. Exemplo de operação do FDMR-MAC.

802.11a/g [Sen, Souvik and Roy Choudhury, Romit and Nelakuditi, Srihari 2011] e o FDMR-MAC fará uso de $\psi(0<\psi<10)$ deles simultaneamente. Assim, o FDMR-MAC pode tirar proveito do OFDMA (Orthogonal frequency-division multiple access) adotando uma política apropriada de alocação de sub-canais para cada uma das $\psi$ transmissões, garantindo um espaçamento entre elas, de modo a possibilitar transmissões simultâneas e evitando interferências. Então, o FDMR-MAC escolhe o sub-canal para o $j$-ésimo nó destino $\left(F_{j}, 0 \leq j \leq \psi\right)$ da seguinte maneira:

$$
F_{j}=j \cdot\left\lceil\frac{\theta}{\psi+1}\right\rceil .
$$

Dessa forma, o FDMR-MAC evita a interferência de sub-canais por vizinhança e torna possível o uso de até $\psi$ sinais pulso/tom simultâneos no canal sem ocorrência de colisão. Considera-se ainda que os nós são capazes de ajustar a potência de transmissão de acordo com o nó vizinho, de modo que a potência recebida pelo nó destino seja próxima a um limiar. Isso permite que um nó identifique que um sinal ou quadro não é direcionado a ele quando a potência do sinal recebido (RSS) seja maior do que o limiar esperado [Mao et al. 2007]. Finalizada esta discussão sobre as características dos sinais pulso e tom, será apresentado um exemplo de comunicação utilizando o FDMR-MAC, de modo a prover uma explicação mais detalhadas sobre a operação do FDMR-MAC.

\subsection{FDMR-MAC: Funcionamento}

Um exemplo do estabelecimento de uma comunicação full-duplex utilizando o FDMRMAC está ilustrado na Figura 1(a). No início, o nó $A$ possui quadros para enviar aos nós $B, C, D$ e $E$, ordenados na fila da camada MAC do nó $A$ como ilustrado na Figura 1(a). Então, o nó $A$ envia um quadro ERTS (Extended Request to Send) para $\psi$ $(1 \leq \psi \leq|\mathcal{X}|)$ nós. No exemplo em questão, considere que $\psi=3$, de modo que o nó 
$A$ envia o quadro ERTS aos nós $B, C$ e $D$. O quadro ERTS possui os mesmos campos contidos no cabeçalho da camada MAC do RTS (Request to Send). No entanto, no quadro ERTS, há a adição de mais $\psi-1$ campos de destino. Ao escutar o quadro ERTS, os nós vizinhos do nó $A$ e que não estão contidos em nenhum dos $\psi$ campos de destino atualizam seu NAV (Network Allocation Vector). O mecanismo NAV é usado para evitar colisões e considera-se o emprego do mesmo mecanismo descrito no padrão IEEE 802.11 [IEEE 2007]. Quando os nós $B, C$ e $D$ recebem o quadro ERTS, cada um deles responde ao nó $A$ com um sinal tone request (tone-r), se possuírem quadros de dados destinados de volta ao nó $A$. Caso não possuam, eles deverão responder com um sinal tone confirm (tone-c) que será posteriormente útil na atualização do NAV.

É importante mencionar que os sinais tone-r e tone-c são enviados de acordo com a política de alocação de sub-canais descrita na Equação (1). Isso ocorre para tornar possível a decodificação dos sinais tone-r e tone-c enviados simultaneamente, sem que sejam percebidas colisões. Portanto, em consonância com a Eq. (1), os nós $B, C$ e $D$ irão enviar os sinais tone-r e tone-c para o nó $A$ utilizando os sub-canais $F_{0}=0, F_{1}=12 \mathrm{e}$ $F_{2}=24$, respectivamente.

Assim, após receber simultâneos sinais tone-r/tone-c como resposta, o nó $A$ pode escolher como destino o nó que respondeu com um tone-r e que está mais bem colocado em sua fila da camada MAC. Caso nenhum nó responda um sinal tone-r (Figura 1(b)), ou seja, todos os nós respondam um sinal tone-c, o nó $A$ escolhe como destinatário da comunicação o nó mais bem posicionado em sua fila da camada MAC. Como ilustrado na Figura 1(a), os nós $C$ e $D$ responderam com um tone-r para o nó $A$. $\mathrm{O}$ nó $C$ foi então escolhido como destinatário por estar mais bem posicionado na fila MAC do nó $A$, como se pode observar na Figura 1(a). Ao escutar os sinais tone-r/tone-c não direcionados a eles, os nós vizinhos dos nós $B, C$ e $D$ estabelecem um pequeno NAV a ser posteriormente confirmado por um quadro de dados ou por um sinal tone-n (tone $N A V$ ), como ocorre nas Figuras 1(a) e 1(b), respectivamente. Este pequeno NAV possui duração igual a $T_{S I F S}+$ $T_{\text {sync }}$, onde $T_{S I F S}$ denota o tempo de SIFS (Short Interframe Space) descrito no padrão IEEE 802.11 [IEEE 2007].

Após o par de nós emissor $(A) / \operatorname{destino}(C)$ ser definido, o nó $A$ envia dados ao nó $C$. Ao escutar este quadro de dados por pelo menos $T_{\text {sync }}$, os nós $B, C$ e $D$ conseguem verificar se o quadro é destinado a eles com base na potência do sinal recebido (RSS), conforme explicado anteriormente. Ao identificar que não foram os escolhidos como destino, os nós $B$ e $D$ estabelecem seu NAV. Por sua vez, o nó $C$ verifica que o quadro de dados é direcionado a ele e começa a mandar seus quadros de dados para o nó $A$, estabelecendo uma comunicação bidirecional. Os vizinhos do nó $C$ escutam estes quadros de dados e estabelecem seu NAV para evitar colisões. Caso o nó destino escolhido $(C)$ não possua quadros de volta para o emissor $(A)$, o nó destino envia um tone-n por pelo menos $T_{\text {sync }}$ para que seus vizinhos possam atualizar seu NAV corretamente. Um exemplo deste cenário onde ocorre o envio de tone-n está ilustrado na Figura 1(b). Em ambos cenários da Figura 1, após receberem os quadros de dados, os nós $A$ e $C$ trocam sinais tone-a (tone acknowledgment) para confirmar o recebimento dos dados.

Note que o FDMR-MAC é capaz de identificar se um sinal é tone-a, tone-c, tone-n ou tone-r, uma vez que estes sinais podem ser diferenciados seja por fase, amplitude ou frequência, conforme descrito por [Liberti and Rappaport 1999]. Para o FDMR-MAC, 
$P_{s z}^{S R} \geq P_{s z}^{R S}$ sempre vale, onde $P_{s z}^{I J}$ denota o tamanho do quadro de dados transmitido do nó $I$ para o nó $J$. Ou seja, FDMR-MAC necessita que o quadro enviado de $R$ para $S$ seja no máximo do tamanho daquele enviado de $S$ para $R$, onde $S$ é o nó emissor que inicia a comunicação enviando o ERTS.

Espera-se que o FDMR-MAC seja um mecanismo eficiente no que concerne à vazão, uma vez que se recorre à utilização de sinais pulso e tom em diversos fases da comunicação (reserva de canal e confirmação de recebimento de quadros dados), reduzindo assim o tempo total de transmissão gasto em uma comunicação. Além disso, o FDMR-MAC aumenta a probabilidade de estabelecer uma comunicação onde os nós emissor e destino possuam quadros de dados para transmitir um ao outro, aumentando assim a vazão e o uso do canal. Os resultados das avaliações realizadas neste trabalho serão apresentados a seguir.

\section{Análise de Resultados}

Essa seção avalia o FDMR-MAC comparando ele com outros esquemas MAC do estado da arte, em especial os protocolos FD-MAC [Zhang et al. 2015] e FDDSMAC [Guimarães and Bordim 2018b]. Tal comparação mostra o impacto do uso do FDMR-MAC na rede, por meio da avaliação em termos de vazão. A avaliação se baseia na métrica definida como sendo o quociente entre a vazão obtida com o FDMR-MAC e a vazão obtida com o outro esquema comparado. Quando a comparação é com o FDMAC, a métrica é denominada $\eta$, enquanto ela é denominada $\gamma$ quando a comparação é com o FDDS-MAC. Assim, se $\eta>1$ e $\gamma>1$, o uso do FDMR-MAC é vantajoso em comparação com o uso do FD-MAC e do FDDS-MAC. Para as avaliações realizadas, o tamanho do quadro de dados $\left(P_{s z}\right)$ variou entre os seguintes valores: 256,512 e 1024 bytes. Além disso, a quantidade de nós vizinhos $(n)$ foi variada de acordo com os seguintes valores: 10 e 50. No que diz respeito aos parâmetros relacionados às camadas física e de controle de acesso ao meio, os valores considerados são aqueles descritos no padrão IEEE 802.11a [IEEE 2007] com uma taxa de transmissão do canal $\left(R_{c}\right)$ igual a 54 Mbps. Embora os parâmetros da avaliação deste trabalho sejam focados no IEEE 802.11a, vale ressaltar que a técnica proposta pode ser aplicada em outras versões dos padrões dos padrões IEEE 802.11.

\subsection{Modelando vazão para comunicações full-duplex}

O cenário considerado nas avaliações realizadas aborda condições de tráfego intenso assumindo que a rede está saturada, isto é, cada nó sempre possui dados para transmitir, fazendo com que todos os nós estejam sempre envolvidos na disputa pelo acesso ao canal. Portanto, um modelo matemático baseado no proposto por Tinnirello et al. [Tinnirello et al. 2010] vai nortear a avaliação realizada. O modelo descrito em [Tinnirello et al. 2010] considera o crescimento do tempo despendido com backoff devido à ocorrência de colisões, o que é fundamental para se possibilitar uma avaliação apropriada do cenário em que se considera a rede como saturada. Sob esta condição, a vazão será denominada vazão de saturação. O modelo apresentado em [Tinnirello et al. 2010] é amplamente utilizado na literatura para avaliar técnicas de controle de acesso ao meio. Diversos autores recorrem a este modelo para realizar suas análises de desempenho, tais como os artigos [Sagari et al. 2015] [Yao et al. 2013] [Nayak et al. 2017] relacionados a comunicações half-duplex. 
Algumas modificações ao modelo podem ser feitas de modo a estendê-lo para o cenário de comunicações full-duplex, como será discutido posteriormente. Logo, o modelo proposto por Tinnirello et al. pode ser utilizado para comparar a vazão de diferentes técnicas de acesso meio em redes em condições de tráfego intenso tanto para técnicas projetadas para comunicações half-duplex quanto para comunicações fullduplex. A definição matemática da vazão de saturação para o referido modelo é a seguinte [Tinnirello et al. 2010]:

$$
S=\frac{p_{s} \cdot \overline{E(P)}}{\left(1-p_{b}\right) \cdot T_{s l o t}+p_{s} \cdot \overline{T_{s}}+\left[p_{b}-p_{s}\right] \cdot \overline{T_{c}}},
$$

onde

$$
\begin{array}{r}
\overline{T_{s}}=T_{s} \cdot \frac{W}{W-1}+T_{\text {slot }}, \\
\overline{T_{c}}=T_{c}+T_{\text {slot }}, \\
\overline{E[P]}=\bar{P}_{s z} \cdot \frac{W}{W-1},
\end{array}
$$

onde $T_{\text {slot }}$ denota o tamanho da fatia de tempo, $T_{s}$ denota o tempo total de transmissão gasto em uma comunicação bem-sucedida, $T_{c}$ denota o tempo despendido em uma colisão de RTS (ou ERTS), $W$ denota o valor mínimo do tamanho da janela de backoff, $p_{s}$ denota a probabilidade da transmissão durante uma fatia de tempo ser bem-sucedida e $p_{b}$ denota a probabilidade de pelo menos um nó transmitir durante uma fatia de tempo. Estas probabilidades $p_{s}$ e $p_{b}$ estão fortemente ligadas aos parâmetros do algoritmo de backoff, tais como os tamanhos mínimo e máximo da janela de backoff. Por limitações de espaço, não haverá maiores explicações destas probabilidades neste trabalho. Para leitores que queiram se aprofundar neste assunto, mais detalhes relacionados ao cálculo de $p_{s}$ e $p_{b}$ estão descritos em detalhes em [Tinnirello et al. 2010].

Existe apenas uma mudança necessária na Eq. (2) para englobar também as comunicações full-duplex. Em comunicações full-duplex bem-sucedidas, o total de dados $\left(P_{s z}\right)$ deve ser a soma dos totais de dados de ambos os sentidos de uma comunicação bidirecional. Esta mudança é necessária pelo fato de que dados podem ser enviados simultaneamente de $S$ para $R$ e de $R$ para $S$, como ilustrado na Figura 1. Assim, para todos os resultados apresentados neste trabalho, o total de dados efetivos transmitidos em uma comunicação full-duplex $\left(\bar{P}_{s z}\right)$ é calculado da seguinte forma:

$$
\bar{P}_{s z}=P_{s z}^{S R}+P_{s z}^{R S} .
$$

Para esta análise, considera-se que o nó $S$ sempre possui dados a enviar ao nó $R$, uma vez que o nó $S$ inicia a comunicação (Figura 1). Além do mais, será considerado que há uma probabilidade $p$ do nó $R$ possuir dados para enviar para o nó $S$. Nas análises apresentadas, essa probabilidade é estabelecida tal que $p=0,1 \cdot x$, onde $1 \leq x \leq 9$. Ademais, será considerado que o tamanho dos dados é igual caso seja estabelecida uma comunicação bidirecional, ou seja, $P_{s z}^{S R}=P_{s z}^{R S}$. Portanto, para os esquemas tradicionais de controle de acesso ao meio tal como o FD-MAC [Zhang et al. 2015], pode-se afirmar que:

$$
\bar{P}_{s z}=P_{s z}^{S R}+p \cdot P_{s z}^{R S}=(1+p) \cdot P_{s z}^{S R} .
$$




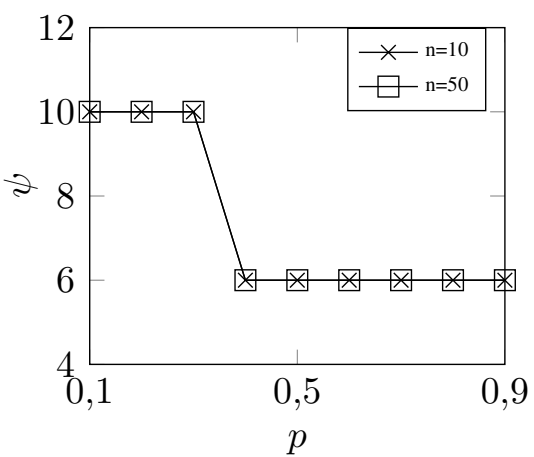

(a) $P_{s z}=256$ bytes, $R_{c}=54 \mathrm{Mbps}$

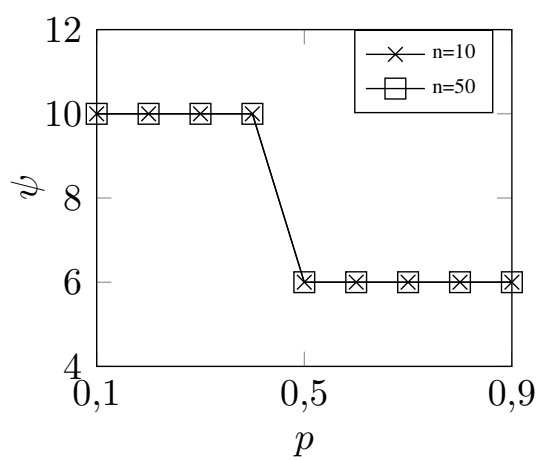

(b) $P_{s z}=1024$ bytes, $R_{c}=54 \mathrm{Mbps}$

Figura 2. Análise de valor ideal de $\psi$.

Por sua vez, o FDMR-MAC possui um valor diferente de $\bar{P}_{s z}$, uma vez que ele tenta estabelecer uma comunicação bidirecional com um dentre os $\psi$ nós destinatários do quadro ERTS. Conforme explicado na Seção 2, no FDMR-MAC, o nó destino precisa de um tempo $\left(T_{\text {sync }}=5 \mu \mathrm{s}\right)$ para confirmar se ele foi realmente o destinatário escolhido antes de começar a enviar dados de volta ao nó emissor. Logo, $P_{s z}^{R S}=P_{s z}^{S R}-k$, onde $k=\left(T_{\text {sync }} \cdot R_{c} / 8\right)$ pois esta é a quantidade em bytes de dados que podem ser enviados no tempo $T_{\text {sync }}$ em um canal com taxa de transmissão igual a $R_{c}$. Portanto, para o FDMRMAC, tem-se que:

$$
\bar{P}_{s z}=P_{s z}^{S R}+\left(1-(1-p)^{\psi}\right) \cdot\left(P_{s z}^{S R}-k\right) .
$$

Para a avaliação do protocolo FDDS-MAC [Guimarães and Bordim 2018b] e o cálculo de sua vazão de saturação, será considerado o valor de $\bar{P}_{s z}$ descrito na Eq. (5) para $k=0$, uma vez que o FDDS-MAC consulta mais de um nó antes de estabelecer sua comunicação de modo análogo ao feito pelo FDMR-MAC. Para todos os mecanismos de controle de acesso ao meio avaliados (FDMR-MAC, FDDS-MAC e FD-MAC), a vazão de saturação será calculada de acordo com a Eq. (2). A seguir, será apresentada uma breve discussão acerca do valor ideal de $\psi$ na operação do FDMR-MAC.

\subsection{Número de destinatários de ERTS}

O valor de $\psi$ influencia diretamente na operação do mecanismo FDMR-MAC. Um alto valor pode onerar a reserva de canal em termos de tempo, uma vez que neste caso o quadro ERTS teria seu tamanho bastante aumentado. Por outro lado, um valor baixo de $\psi$ pode não aumentar muito a probabilidade de estabelecer comunicações bidirecionais a um custo de estender o RTS para o ERTS. Neste contexto, foi realizada uma avaliação empírica a respeito do valor de $\eta$ para várias combinações de valores de $\psi(2<\psi<10)$, $n, P_{s z}$ e $p$. Então, o valor de $\psi$ que obteve o maior $\eta$ para uma determinada combinação de $n, P_{s z}$ e $p$ é selecionado como sendo o $\psi$ ideal para aquela combinação. Os valores ideais de $\psi$ para uma taxa de transmissão de $54 \mathrm{Mbps}$ estão retratados na Figura 2. Nesta figura, os valores obtidos como ideais de $\psi$ para quando $P_{s z}=512$ bytes não foram apresentados por limitações de espaço e por serem similares aos demais apresentados na Figura 2. Como pode ser observado nas Figuras 2(a) e 2(b), os valores ideais de $\psi$ aumentam à medida que $p$ diminui. A análise do valor ideal de $\psi$ irá focar nos valores intermediários 
de probabilidade $p$. Para $R_{c}=54 \mathrm{Mbps}$, nota-se que o valor ideal de $\psi$ só possui dois valores selecionados: $\psi=6$ para valores maiores de $p$ e $\psi=10$ para valores menores de $p$, conforme retratado nas Figuras 2(a) e 2(b). Este comportamento será explicado a seguir. É importante relembrar que o crescimento de $\psi$ aumenta o tamanho do cabeçalho do quadro ERTS, aumentando assim o tempo total para transmiti-lo. Como descrito no padrão IEEE 802.11a [IEEE 2007], o tempo total de transmissão para enviar um quadro RTS $\left(T_{R T S}\right)$ é definido da seguinte forma:

$$
T_{R T S}=20+4 \cdot\left\lceil\left(16+6+8 \cdot H_{s z}\right) /(4 \cdot 54)\right\rceil,
$$

onde $H_{s z}$ denota o tamanho do cabeçalho do RTS em bytes. Tipicamente, $H_{s z}=20$. Para o quadro ERTS, $H_{s z}$ é aumentado por possuir mais $\psi-1$ campos destino que o RTS. É importante mencionar que cada campo destino possui 6 bytes. Logo, o tempo gasto para enviar um quadro ERTS é tal que:

$$
\left.T_{\text {ERTS }}=20+4 \cdot\lceil(16+6+8 \cdot(20+6 \cdot(\psi-1))) / 4 \cdot 54)\right\rceil .
$$

Quando $\psi \in[2,6]$, pode-se notar que $T_{E R T S}=28 \mu \mathrm{s}$. Portanto, para uma taxa de transmissão do canal de $54 \mathrm{Mbps}$, o tempo gasto para enviar um ERTS com 2 ou 6 campos de destino será o mesmo. Assim, o $\psi$ que maximiza $\eta$ será 6 neste caso, uma vez que a probabilidade de se estabelecer uma comunicação bidirecional aumenta na medida que $\psi$ cresce, conforme indicado na Eq (5). Um argumento semelhante vale para o intervalo $\psi \in[7,10]$, uma vez que nesse caso $T_{E R T S}$ é igual a $32 \mu s$, o que faz com que o $\psi$ ideal seja igual a 10 para os valores menores de $p$ na Figura 2. Como a análise será focada nos valores intermediários de $p$, considera-se que para $R_{c}=54 \mathrm{Mbps}, \psi=6$ será o valor escolhido para as avaliações subsequentes. Terminada esta discussão e avaliação acerca do valor de $\psi$, uma análise comparativa envolvendo FDMR-MAC, FDDS-MAC e FD-MAC em termos de vazão de saturação será apresentada a seguir.

\subsection{Vazão de Saturação}

Uma comparação entre FDMR-MAC e FD-MAC [Zhang et al. 2015] em termos de $\eta$ está disponível na Figura 3. $\eta>1$ ocorre em todos os casos avaliados, indicando portanto que o FDMR-MAC provê uma maior vazão de saturação do que o FD-MAC. $\eta$ chega a alcançar até $1,67,1,66$ e 1,62 quando $P_{s z}=256, P_{s z}=512$ e $P_{s z}=1024$ bytes, respectivamente. É importante mencionar que $\eta$ obteve seus maiores valores quando $p=$ 0,2 e $n=10$. Para $P_{s z}=256$ bytes, $\eta$ foi em média igual a 1,44 e foi o seu valor mínimo foi $1,15(\eta>=1,15)$. Por sua vez, quando $P_{s z}=512$ bytes, o valor mínimo foi de 1,16 $(\eta>=1,16)$, possuindo média novamente igual a 1,44. Para $P_{s z}=1024$ bytes, o valor médio de $\eta$ é igual a 1,42 e o mínimo é de 1,15 ( $\eta>=1,15)$. Portanto, pode-se inferir que o FDMR-MAC pode melhorar a vazão de saturação significativamente quado comparado com o FD-MAC, uma vez que obteve-se $\eta>1$ para todos os casos avaliados, chegando a alcançar um ganho de até $67 \%(\eta=1,67)$.

Algumas tendências ocorreram para os três casos. Como pode-se observar na Figura 3, à medida que $n$ aumenta, $\eta$ diminui. Isso pode ser explicado pelo fato de que quando $n$ aumenta, $T_{c}$ tende a ser mais significativo no cálculo da vazão de saturação $S$ (Eq. (2)). Além disso, $T_{c}$ para o FD-MAC é menor do que para o FDMR-MAC, uma vez 

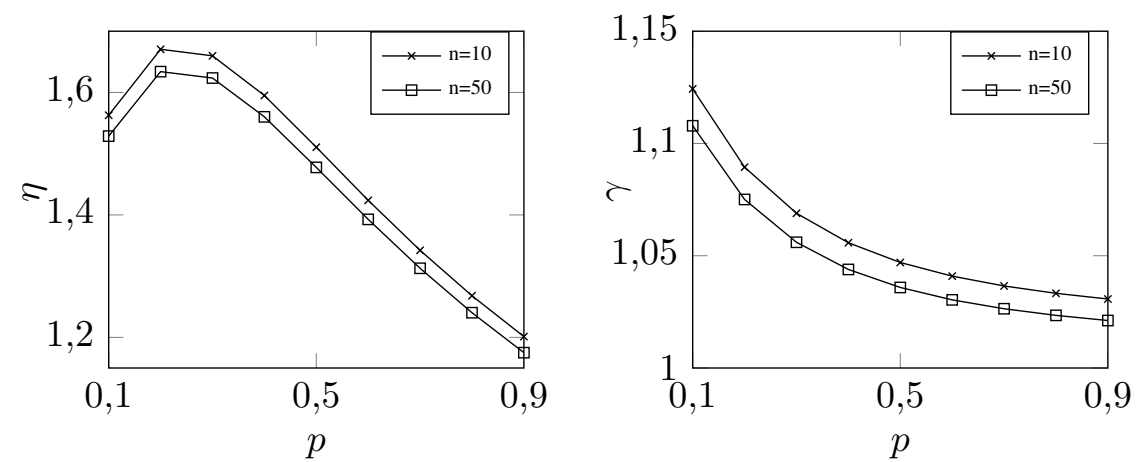

(a) $P_{s z}=256$ bytes, $R_{c}=54 \mathrm{Mbps}$

(b) $P_{s z}=256$ bytes, $R_{c}=54 \mathrm{Mbps}$
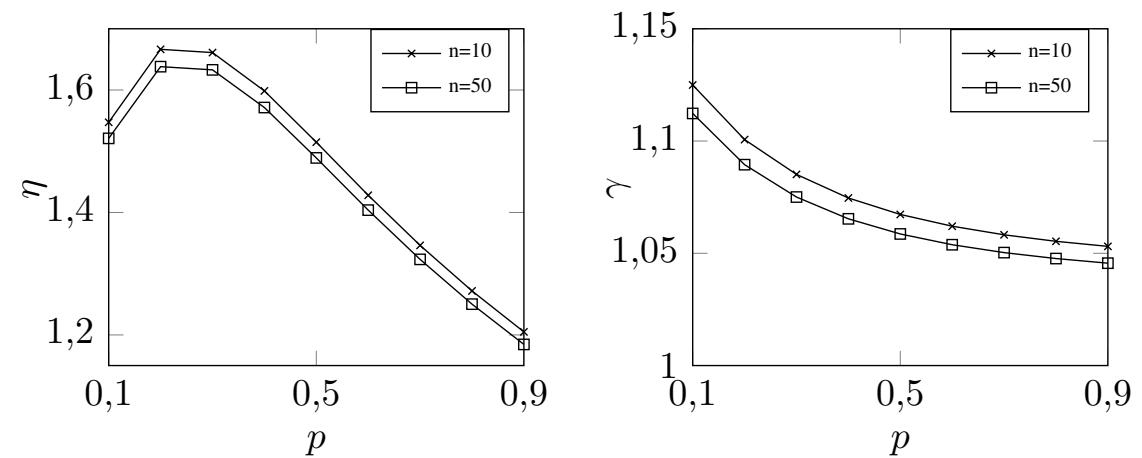

(c) $P_{s z}=512$ bytes, $R_{c}=54 \mathrm{Mbps}$

(d) $P_{s z}=512$ bytes, $R_{c}=54 \mathrm{Mbps}$
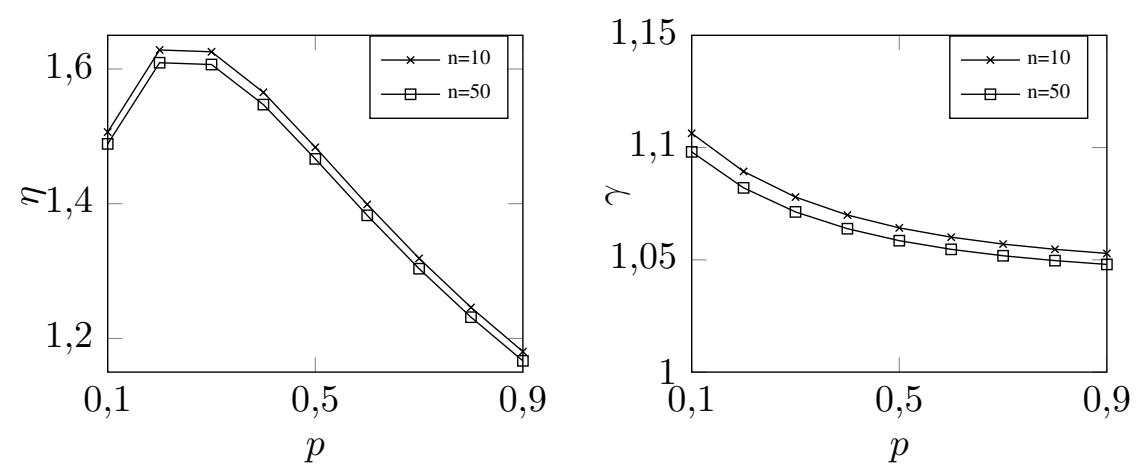

(e) $P_{s z}=1024$ bytes, $R_{c}=54 \mathrm{Mbps}$

(f) $P_{s z}=1024$ bytes, $R_{c}=54 \mathrm{Mbps}$

Figura 3. Valores de $\eta \mathbf{e} \gamma$ para diversos valores de $p$, comparando FDMR-MAC com FD-MAC e FDDS-MAC. 
Tabela 1. Valores calculados de $\gamma$ para diferentes cenários, comparando FDMRMAC com FDDS-MAC.

\begin{tabular}{|c|c|c|c|c|c|c|}
\cline { 4 - 7 } \multicolumn{2}{c|}{$\gamma$} & \multicolumn{4}{c|}{$\gamma$} \\
\hline$R_{c}$ & $P_{s z}$ & $p$ & $n$ & Máximo & Média & Mínimo \\
\hline $54 \mathrm{Mbps}$ & 256 bytes & 0,1 & 10 & 1,12 & 1,05 & 1,01 \\
$54 \mathrm{Mbps}$ & 512 bytes & 0,1 & 10 & 1,12 & 1,07 & 1,04 \\
$54 \mathrm{Mbps}$ & 1024 bytes & 0,1 & 10 & 1,11 & 1,06 & 1,04 \\
\hline
\end{tabular}

que o quadro RTS é menor do que o quadro ERTS. Isso ocorre pelo fato do ERTS possuir $\psi-1$ campos de destino a mais do que o RTS para tentar aumentar a probabilidade de que os dois nós envolvidos na comunicação transmitam dados um ao outro simultaneamente, estabelecendo uma comunicação bidirecional. Apesar disso, é notável que o FDMR-MAC melhorou a vazão de saturação significativamente. Os ganhos obtidos devido ao eficiente mecanismo de reserva de canal do FDMR-MAC compensaram o tempo perdido com o aumento do quadro ERTS. Além do mais, é importante mencionar que a adição de $\psi-1$ campos de destino no ERTS pode não ser tão custosa em termos de tempo, quando a taxa de transmissão do canal $\left(R_{c}\right)$ é igual a $54 \mathrm{Mbps}$. O aumento de $\psi$ sem comprometer muito o tempo total de transmissão também favorece uma melhoria na vazão de saturação, uma vez que um valor maior de $\psi$ deve aumentar a quantidade esperada de dados transmitidos, como pode ser inferido da Eq. (5).

Para realizar uma avaliação mais abrangente, o FDMR-MAC também foi comparado com o FDDS-MAC [Guimarães and Bordim 2018b] em termos de vazão de saturação. Os resultados dessa avaliação estão disponíveis na Figura 3. Um resumo destes resultados se encontra apresentado na Tabela 1. Esta tabela apresenta os valores máximo, mínimo e médio de $\gamma$ para diferentes valores de $P_{s z}$. Além disso, a Tabela 1 mostra os valores de $p$ e $n$ que maximizaram $\gamma$ para um determinado $P_{s z}$. É notável que $\gamma>1$ em todos os casos avaliados, isto é, o FDMR-MAC superou o FDDS-MAC em termos de vazão de saturação para todos os casos avaliados. É importante lembrar que o FDDS-MAC possui uma política de escalonamento que favorece o estabelecimento de comunicações bidirecionais em uma estratégia similar à proposta pelo FDMR-MAC. Por isso, esperava-se que a melhoria obtida com o FDMR-MAC fosse maior em relação ao FD-MAC do que em relação ao $\operatorname{FDDS}-M A C(\eta>\gamma>1)$, o que de fato se concretizou nas análises realizadas. Como o processo de reserva de canal e escolha do nó destino do FDMR-MAC gasta menos tempo que o do FDDS-MAC, o FDMR-MAC consegue atingir uma melhoria de vazão de saturação de até $12 \%$ em relação ao FDDS-MAC.

Os resultados apresentados reforçaram a expectativa acerca do impacto positivo da utilização do FDMR-MAC em comunicações full-duplex, uma vez que o FDMR-MAC obtém ganhos de vazão de saturação de até $67 \%$ em relação ao FD-MAC e de até $12 \%$ em relação ao FDDS-MAC. Este argumento encerra a análise do impacto do FDMR-MAC sobre as comunicações full-duplex.

\section{Conclusão}

O uso eficiente do canal é fundamental no contexto da quinta geração (5G) de redes móveis. Portanto, este trabalho propõe o FDMR-MAC que é uma técnica de controle de acesso ao meio projetada para comunicações full-duplex capaz de melhorar o uso do 
espectro, com um mecanismo inovador de reserva de canal baseado em sinais pulso e tom em conjunto com adaptação do quadro RTS.

Para avaliar o FDMR-MAC, o modelo matemático proposto por Tinnirello et al. [Tinnirello et al. 2010] foi estendido para abordar também as comunicações fullduplex. O FDMR-MAC foi comparado com dois protocolos de controle de acesso ao meio do estado da arte projetados para comunicações full-duplex. Os protocolos em questão (FD-MAC e FDDS-MAC) possuem diferentes estratégias no que diz respeito à reserva de canal e políticas de escalonamento. As comparações realizadas em diversos cenários indicam que o FDMR-MAC superou ambos os protocolos em todos os casos avaliados. O ganho do FDMR-MAC sobre o FD-MAC e o FDDS-MAC alcança até $67 \%$ e $12 \%$, respectivamente. Portanto, fica evidente a contribuição e relevância do FDMR-MAC no contexto de técnicas de controle de acesso ao meio projetadas para comunicações fullduplex.

No que diz respeito a trabalhos futuros, seria interessante incorporar ao FDMRMAC algum mecanismo capaz de estimar com precisão os valores de $P_{s z}, n$ e $p$ de maneira dinâmica. Caso tais valores fossem conhecidos previamente ao início de cada comunicação, um nó poderia decidir de modo apropriado qual o melhor valor de $\psi$ $(0<\psi<10)$ a ser usado na operação do FDMR-MAC, de modo a maximizar o uso do canal.

\section{Agradecimentos}

Este trabalho foi parcialmente financiado por MCTIC/RNP/CTIC através do projeto P4Sec.

\section{Referências}

Al-Kadri, M. O., Aijaz, A., and Nallanathan, A. (2016). An energy-efficient full-duplex MAC protocol for distributed wireless networks. IEEE Wireless Communications Letters, 5(1):44-47.

Amjad, M., Akhtar, F., Rehmani, M. H., Reisslein, M., and Umer, T. (2017). Full-duplex communication in cognitive radio networks: A survey. IEEE Communications Surveys \& Tutorials.

Garcia-Luna-Aceves, JJ (2017). Carrier-Sense Multiple Access with Collision Avoidance and Detection. In Proceedings of the 20th ACM International Conference on Modelling, Analysis and Simulation of Wireless and Mobile Systems, pages 53-61. ACM.

Guimarães, L. D. M. and Bordim, J. L. (2018a). A Full-Duplex MAC Tailored for 5G Wireless Networks. Wireless Communications and Mobile Computing, 2018.

Guimarães, L. D. M. and Bordim, J. L. (2018b). FDDS-MAC: Enhancing spectrum usage on full-duplex communications in 5G mobile wireless networks. In IEEE Symposium on Computers and Communications (ISCC), pages 268-273. IEEE.

Guimarães, L. D. M., Bordim, J. L., and Nakano, K. (2015). Using pulse/tone signals as an alternative to boost channel reservation on directional communications. IEICE TRANSACTIONS on Fundamentals of Electronics, Communications and Computer Sciences, 98(8):1647-1656. 
IEEE (2007). IEEE Standard for information technology-telecommunications and information exchange between systems-local and metropolitan area networks - specific requirements - part 11: Wireless lan medium access control (mac) and physical layer (phy) specifications. IEEE Standard 802.11, Institute of Electrical and Electronics Engineers.

Liberti, J. and Rappaport, T. (1999). Smart antennas for wireless communications. Prentice Hall PTR.

Mao, G., Anderson, B. D. O., and Fidan, B. (2007). Path loss exponent estimation for wireless sensor network localization. Computer Networks, 51(10):2467-2483.

Marlali, Deniz and Gurbuz, Ozgur (2017). Design and performance analysis of a fullduplex MAC protocol for wireless local area networks. Ad Hoc Networks, 67:53-67.

Nayak, P., Garetto, M., and Knightly, E. W. (2017). Multi-user downlink with single-user uplink can starve TCP. In INFOCOM 2017-IEEE Conference on Computer Communications, IEEE, pages 1-9. IEEE.

Sagari, S., Baysting, S., Saha, D., Seskar, I., Trappe, W., and Raychaudhuri, D. (2015). Coordinated dynamic spectrum management of LTE-U and Wi-Fi networks. In Dynamic Spectrum Access Networks (DySPAN), 2015 IEEE International Symposium on, pages 209-220. IEEE.

Sen, Souvik and Roy Choudhury, Romit and Nelakuditi, Srihari (2011). No time to countdown: Migrating backoff to the frequency domain. In Proceedings of the 17th annual international conference on Mobile computing and networking, pages 241-252. ACM.

Shih, K., Liao, W., Chen, H., and Chou, C. (2009). On avoiding RTS collisions for IEEE 802.11-based wireless ad hoc networks. Computer Communications, 32(1):69-77.

Tang, R., Zhao, J., Qu, H., and Zhang, Z. (2016). Energy-efficient resource allocation for 5G full-duplex enabled device-to-device communication. In Globecom Workshops (GC Wkshps), 2016 IEEE, pages 1-7. IEEE.

Thilina, K. M., Tabassum, H., Hossain, E., and Kim, D. I. (2015). Medium access control design for full duplex wireless systems: challenges and approaches. IEEE Communications Magazine, 53(5):112-120.

Tinnirello, I., Bianchi, G., and Xiao, Y. (2010). Refinements on IEEE 802.11 distributed coordination function modeling approaches. IEEE Transactions on Vehicular Technology, 59(3):1055-1067.

Yao, Y., Rao, L., and Liu, X. (2013). Performance and reliability analysis of IEEE 802.11p safety communication in a highway environment. IEEE transactions on vehicular technology, 62(9):4198-4212.

Zhang, X., Cheng, W., and Zhang, H. (2015). Full-duplex transmission in PHY and MAC layers for 5G mobile wireless networks. IEEE Wireless Communications, 22(5):112121.

Zhang, Z., Long, K., Vasilakos, A. V., and Hanzo, L. (2016). Full-duplex wireless communications: challenges, solutions, and future research directions. Proceedings of the IEEE, 104(7):1369-1409. 